\title{
Effect of antibodies binding to Y chromosome- bearing sperm conjugated with magnetic nanoparticles on bull sperm characteristics
}

\author{
So-Yeon Jo ${ }^{1}$, Yong Hwangbo ${ }^{1}$, Sang-Hee Lee ${ }^{1}$, Hee-Tae Cheong ${ }^{2}$, Dong-Ku Kim ${ }^{3}$ and \\ Choon-Keun Park ${ }^{1, *}$ \\ ${ }^{1}$ College of Animal Life Sciences, Kangwon National University, Chuncheon 24341, Korea \\ ${ }^{2}$ College of Veterinary Medicine, Kangwon National University, Chuncheon 24341, Korea \\ ${ }^{3}$ Nuriscience Inc, Seoul 05053, Korea
}

Received November 16, 2021

Revised December 7, 2021

Accepted December 9, 2021

\section{*Correspondence \\ Choon-Keun Park \\ E-mail: parkck@kangwon.ac.kr}

Author's Position and Orcid no. Jo SY, MS student,

https://orcid.org/0000-0002-3751-457X

Hwangbo $\mathrm{Y}, \mathrm{PhD}$ candidate,

https://orcid.org/0000-0003-3636-3551

Lee $\mathrm{SH}$, Professor,

https://orcid.org/0000-0001-8725-4174

Cheong HT, Professor,

https://orcid.org/0000-0001-8225-0262

Kim DK, PhD,

https://orcid.org/0000-0002-7633-4504

Park CK, Professor,

https://orcid.org/0000-0003-2786-8814

\begin{abstract}
The immunological sperm separation method is economical compared to the existing sorting method, and it is promising for the development of new technologies by reducing sperm damage. Wholemom (WM) is a sex-regulating protein that comprises on immunoglobulin G coupled with magnetic nanoparticles (MNPS) that responds to surface proteins derived from the $Y$ chromosome in cattle. $Y$ sperms are restricted in motility as the WM aggregates them, and the magnet could separate the non-aggregated cells. This study was conducted to investigate the effect of WM treatment on the characteristics of bull sperm. After treating sperm with WM and incubation for $6 \mathrm{~h}$, the motility parameters including total motility, progressive motility, velocity average path, velocity straight line, amplitude of lateral head displacement, and linearity were significantly higher in the WM treatment group than in the control group ( $p<0.05)$. Sperm viability and acrosome reaction rates were similar in both groups during each incubation period $(p>0.05)$. In conclusion, the immunological sperm sexing procedure using a monoclonal antibody conjugated with MNPs did not affect the characteristics of bull sperm. This study suggests that compared to other techniques, the immunological method for sperm sexing could classify sperm quickly and efficiently without the use of expensive equipment.
\end{abstract}

Keywords: immunological method, sperm characteristics, sperm sexing, Y specific antibody

\section{INTRODUCTION}

Production of offspring of the desired sex by artificial regulation is considered critical to improve livestock productivity and increase economic efficiency in the livestock industry. Dairy experts consider dairy products very important from a food security and nutritional perspective (Al-Dulaimi and Al-Timimi, 2018; Safari et al.,
2018). In addition, rapid economic growth and population growth have led to the expansion of livestock product consumption and the securing of various distribution channels.

In mammals, the sex of the offspring is determined by the sex chromosomes in the sperm. Altering the normal 1:1 ratio of $X$ or $Y$ sperm to favor conception of one sex or the other is the most logical approach to control the sex ratio. 
The artificial sex-separation of spermatozoa is representative of reproductive biotechnology for livestock industry species. These techniques are based on the physiological differences in $\mathrm{X}$ - or $\mathrm{Y}$-bearing sperm. In cattle, $\mathrm{X}$ chromosomebearing sperm contains approximately $4 \%$ more DNA than $Y$ chromosome-bearing sperm (Garner et al., 1983; Johnson and Clarke, 1988), and $Y$ sperms swim faster than $X$ sperms (Penfold et al., 1998). In addition, other differences include the size (Cui, 1997), and surface charge on sperm (Kaneko et al., 1983). Several studies have shown that flow cytometric measurement of DNA content in individual sperm allows the resolution of $X$ and $Y$ populations (Seidel Jr, 2012; Laxmivandana et al., 2021). Other commercially used methods of sorting $X$ - or $Y$-sperms have been reported, including swim-up (Han et al., 1993), $\mathrm{H}-\mathrm{Y}$ antigen (Bennett and Boyse, 1973), and percoll gradient centrifugation (Koundouros and Verma, 2012), that based on physiological differences. However, the sperm separation methods developed to date have resulted in significantly reduced sperm motility and viability owing to the isolation process (laser, high pressure, fluorescence, and electrical stimulation) despite the high cost (Seidel Jr, 2012). In addition, the sperm concentration of the separated semen is reduced compared to that of conventional semen and this reduction could lower the pregnancy rate of livestock, causing economic loss to farmers. These limitations have prompted the establishment of efficient and non-invasive approaches for sperm sorting.

Across species, the observed differences in DNA between $\mathrm{X}$ and Y-bearing sperm led to the possibility that these differences might result in protein differences. Based on this concept, immunological sex separation methods for sperm using different proteins present on the surface of X- and Y-bearing sperm have been developed. The $\mathrm{H}-\mathrm{Y}$ antigen is a male-specific antigen on the sperm surface and is separated from the sex-determining region $\mathrm{Y}$ (SRY). It has been reported that the $\mathrm{H}-\mathrm{Y}$ antigen preferentially binds the anti-H-Y antibody to $\mathrm{Y}$ sperms (Zavos, 1983), enabling the identification and isolation of sperm using the $\mathrm{H}-\mathrm{Y}$ antigen. However, there was no change in the sex ratio of the offspring even after using this antibody, and Prasad et al. (2010) reported that the H-Y antigen was present in the cell membranes of both $\mathrm{X}$ - and Y-bearing sperm. Therefore, further research is needed to isolate semen using the $\mathrm{H}-\mathrm{Y}$ antigen. A proteomics approach has been developed based on the protein profile differentially expressed in $\mathrm{X}$ - and Y-bearing sperm (De
Canio et al., 2014). It has been shown that sex-dependent protein expression in cattle can affect sperm function, phenotype, and interactions between sperm and oocytes (Li et al., 2016). These proteins could be used as potential molecular markers to differentiate and classify $\mathrm{X}^{-}$and Y-bearing sperm. Sperm sorting based on immunological methods is a reproducible, economic and convenient approach. Immunological methods are promising for the development of new technologies to overcome existing limitations, and are expected to benefit the agricultural industry.

Wholemom (WM) is sex-regulating protein comprising an immunoglobulin $G$ coupled with magnetic nanoparticles (MNPs) that responds to surface proteins derived from the Y chromosome in cattle. The WM binds to the plasma membrane of Y-bearing sperm, and the agglutinated sperm settles on the bottom or is fixed with a magnet. This technique aims to produce female individuals by allowing only $\mathrm{X}$-bearing sperm to migrate to the fertilization site without loss in motility. This study hypothesized that a method based on the physiological differences of sperm with $\mathrm{X}$ and $\mathrm{Y}$ would lead to efficient sperm isolation. This study was conducted to investigate the effect of WM treatment on the characteristics of bull sperm.

\section{MATERIALS AND METHODS}

\section{Chemicals}

All reagents used in this study were obtained from Sigma Chemical Co. (St Louis, MO, USA) unless otherwise indicated.

\section{Preparation of sexed sperm}

Frozen semen from Korean native cattle (Hanwoo) was obtained from the Hanwoo Improvement Center (NongHyup Agribusiness Group Inc., Chungcheongnam-do, Korea). Eight frozen semen straw of same bull were used for each replication and 3 to 5 animals were used in each experiment. One frozen semen straw was thawed at $37^{\circ} \mathrm{C}$ for $1 \mathrm{~min}$. Then, the sperm were suspended in $5.0 \mathrm{~mL}$ of $\mathrm{KO}$ solution (sperm washing medium; $0.105 \mathrm{~g} / \mathrm{L}$ magnesium chloride hexahydrate, $0.249 \mathrm{~g} / \mathrm{L}$ calcium chloride, $4.68 \mathrm{~g} /$ $\mathrm{L}$ sodium chloride, $0.3 \mathrm{~g} / \mathrm{L}$ potassium chloride, $0.129 \mathrm{~g} / \mathrm{L}$ sodium phosphate monobasic dihydrate, $5.96 \mathrm{~g} / \mathrm{L}$ HEPES, $2.518 \mathrm{~g} / \mathrm{L}$ D-glucose, $0.06 \mathrm{~g} / \mathrm{L}$ L-cystine, $1.06 \mathrm{~g} / \mathrm{L}$ caffeine, $8.0 \mathrm{mg} / \mathrm{L}$ phenol red, $3.108 \mathrm{~g} / \mathrm{L}$ sodium bicarbon- 
ate) containing $0.5 \%$ BSA. Both the control group and the WM treatment group were prepared using the same washing media.

Sperm motility was visually confirmed under a stereoscopic microscope and the solution was centrifuged at $430 \times \mathrm{g}$ for $5 \mathrm{~min}$. After removing the supernatant including the cryopreservation solution, $1.0 \mathrm{~mL}$ of $\mathrm{KO}$ solution and 1 vial of WM (Nuri Science, Gyeonggi-do, Korea) was added to the sperm pellet and allowed to stand at $37^{\circ} \mathrm{C}$ for 20 min. Because the MNPs can sink, the tube containing the sperm treated with WM was periodically rotated to react with the target cells of the WM antibody. After $20 \mathrm{~min}$, the WM-treated sperm were transferred to a $5.0 \mathrm{~mL}$ round tube surrounded by a magnet (STEMCELL, CA-BC, Canada), and KO solution was added so that the total volume was $3.0 \mathrm{~mL}$. After reacting for $5 \mathrm{~min}$ under a magnetic environment, $1.0 \mathrm{~mL}$ of the supernatant was carefully recovered using a pipette so as to not touch the sperm aggregated on the wall of the round tube. The transferred sperm were centrifuged at $430 \times \mathrm{g}$ for $5 \mathrm{~min}$ with $\mathrm{KO}$ solution and the sperm was used to analyze sperm characteristics. Control sperm were centrifuged twice times during the preparation of the WM treatment group and used in each experiment.

\section{Computer-assisted sperm analysis (CASA)}

Sperm motility was assessed using the CASA system (Hamilton Thorne, Beverly MA, USA). This program consists of a phase-contrast microscope, a digital camera, a thermal stage warmer, and a computer that stores and analyzes the data. For approximately 200 sperm sample, sperm motility was measured at $0,2,4$, and 6 h under $100 \times$ magnification. Ten microliters of sperm diluted to a concentration of $1.0 \times 10^{6}$ sperm $/ \mathrm{mL}$ was mounted in a Standard Count 4 Chamber Slide (IMV Technologies, L' Aigle, France), and microscopic fields of five randomly selected sections were scanned and used for statistical analysis. Nine sperm parameters including total motility (TM; \%), progressive motility (PM; \%), velocity average path (VAP; $\mu \mathrm{m} / \mathrm{s})$, velocity straight line (VSL; $\mu \mathrm{m} / \mathrm{s})$, curvilinear velocity (VCL; $\mu \mathrm{m} / \mathrm{s})$, the amplitude of lateral head displacement (ALH; $\mu \mathrm{m})$, beat-cross frequency (BCF: Hz), straightness (STR; \%), and linearity (LIN; \%) were analyzed using CASA program.

\section{Fluorescent staining and flow cytometry analysis}

Flow cytometry analysis was performed using a FACSCalibur flow cytometer (Becton Dickinson, NJ, USA). Sexseparated sperm presumed to be $\mathrm{X}$ chromosomes were centrifuged for $5 \mathrm{~min}$ at $430 \times \mathrm{g}$, and then, the supernatant was removed. Sperm samples were resuspended to be $1.0 \times 10^{6} \mathrm{sperm} / \mathrm{mL}$ with $1.0 \mathrm{~mL}$ of PBS (-), and sperm viability and acrosome integrity were analyzed using a fluorescent dye.

A LIVE/DEAD Sperm Viability Kit (L7011, Molecular Probes, CA, USA) was used to detect sperm viability. The final concentration of SYBR-14 was adjusted to $0.78 \mathrm{nM}$ in diluted sperm samples and incubated for $5 \mathrm{~min}$ in a dark room at $38^{\circ} \mathrm{C}$. Then, propidium iodide (PI) was added to be $37.5 \mathrm{nM}$ in the samples and cultured under the same conditions. After staining, the sperm were centrifuged for $5 \mathrm{~min}$ at $430 \times \mathrm{g}$. The supernatant was removed and resuspended in $300 \mu \mathrm{L}$ PBS (-). A total of 10,000 cells were analyzed by flow cytometry. Flow cytometry data were analyzed using Flowing Software (Version 2.5.1, Turku, Finland).

The acrosome integrity of sperm was evaluated after staining the sperm with fluorescein isothiocyanateconjugated peanut agglutinin (FITC-PNA; L7381) and PI double staining. The FITC-PNA $(2.5 \mu \mathrm{g} / \mathrm{mL})$ was added to the sperm samples and incubated for $5 \mathrm{~min}$ in a dark room at $38^{\circ} \mathrm{C}$. Then, PI was added to sperm samples until $37.5 \mathrm{nM}$ and cultured under the same conditions. After removing the supernatant, $300 \mu \mathrm{L}$ of PBS (-) was added. A total 10,000 sperms were analyzed with flow cytometry and data were analyzed with Flowing Software.

Bull sperm were stained and examined using a fluorescence microscope for visual confirmation. To determine the viability of sperm, sperm samples were diluted to 5.0 $\times 10^{6} \mathrm{sperm} / \mathrm{mL}$ in $1.0 \mathrm{~mL}$ of PBS (-). $1.0 \mu \mathrm{L}$ of SYBR-14 $(1.0 \mathrm{mM}, \mathrm{v} / \mathrm{v})$ and $2.5 \mu \mathrm{L}$ of PI $(2.4 \mathrm{mM}, \mathrm{v} / \mathrm{v})$ were mixed with sperm samples and incubated at $38^{\circ} \mathrm{C}$ in a dark room for $5 \mathrm{~min}$. After staining, the samples were centrifuged for $5 \mathrm{~min}$ at $430 \times \mathrm{g}$, and the sperm pellet was resuspended in $100 \mu \mathrm{L}$ of PBS (-). Acrosome integrity of sperm was confirmed by staining $1.0 \mu \mathrm{L}$ of FITC-PNA $(5 \mathrm{mg} / \mathrm{mL}$, v/ v) and $1.0 \mu \mathrm{L}$ of Hoechst $33342(1 \mathrm{mg} / \mathrm{mL}, \mathrm{v} / \mathrm{v})$ in $1.0 \mathrm{~mL}$ of PBS (-) containing $5.0 \times 10^{6} \mathrm{sperm} / \mathrm{mL}$ of cells. After incubation under the same conditions and centrifugation, the pellet was resuspended in $100 \mu \mathrm{L}$ of PBS (-). A labeled $10 \mu \mathrm{L}$ sperm sample was placed on a microscope 
slide and covered with a cover glass. Slides were observed and recorded under a fluorescence microscope (Olympus BX 50; Olympus, Tokyo, Japan) at $400 \times$ magnification. Live sperm were stained green by SYBR-14 whereas the membrane-damaged sperm were stained red with PI. Entire sperm were stained with Hoechst 33342 as blue fluorescence, and acrosome-reacted sperm were stained with FITC-PNA as green fluorescence.

\section{Statistical analysis}

The data were analyzed using Statistical Analysis System Software (SAS; version 9.4, NC, USA) and expressed as mean \pm standard error of the mean (SEM). When the data were percentages, we arcsine transformed the data to improve the approximation to normality. A $p$-value of $<0.05$, was considered indicative of a statistically significant difference. Differences between groups were assessed using the Student's $t$-test followed by Duncan's multiple range test using one-way analysis of variance (ANOVA).

\section{RESULTS}

\section{Effect of WM treatment on the agglutination of bull sperm}

Sperm in the upper layer presumed to have an X chromosome did not exhibit aggregation, however, it was vi- sually confirmed that the sperm aggregates and attached to the magnet by WM were head-to-head aggregation. Aggregated sperm are presumed to be cells with a Y chromosome, and it was observed that they had less motility than sperms in the supernatant owing to WM treatment (Fig. 1).

\section{Effect of WM treatment on the characteristics of bull sperm}

The motility parameters recorded during incubation after the treatment of sperm with WM are shown in Table 1. The TM, PM, and VCL were reduced in both sperm groups after $2 \mathrm{~h}$. After $6 \mathrm{~h}$ of incubation, all parameters, except VCL, BCF, and STR were significantly higher in the WM treatment group than in the control group $(p<0.05)$. No difference was observed in BCF and STR, regardless of WM treatment during the incubation period. Sperm viability and acrosome reaction rates did not differ between the both sperm groups at each incubation time (Fig. 2).

\section{DISCUSSION}

The purpose of this study was to analyze changes in the motility, viability, and acrosome damage of sex-separated bull sperm by treatment with WM, a protein that regulates sex. The WM treatment group showed higher motility
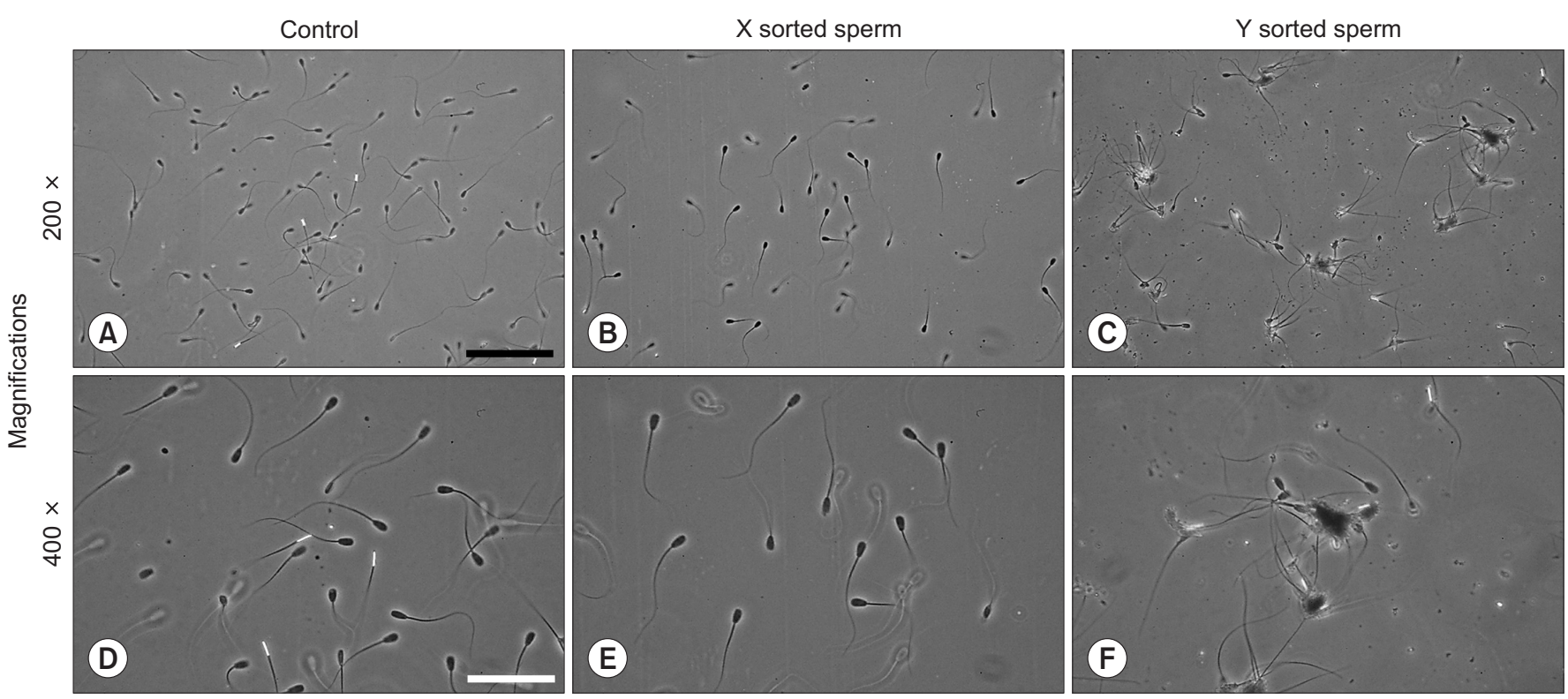

Fig. 1. Morphology of agglutinated spermatozoa of Korean native cattle (Hanwoo) at 25 min after Wholemom (WM) treatment, pretreatment of bull sperm ( $A, D)$, non-agglutinated sperm ( $B, E$; assumed $X$-sorted sperm), and agglutinated sperm with WM ( $C$, $F$; assumed Y-sorted sperm), black scale bar: $400 \mu \mathrm{m}$, white scale bar: $200 \mu \mathrm{m}$. 
Table 1. Computer-assisted sperm analysis (CASA) of bull sperm during different incubation time with or without Wholemom (WM) treatment

\begin{tabular}{|c|c|c|c|c|c|}
\hline \multirow{2}{*}{ Parameters } & \multirow{2}{*}{ Treatment group } & \multicolumn{4}{|c|}{ Period of sperm incubation time (hours) } \\
\hline & & 0 & 2 & 4 & 6 \\
\hline \multirow[t]{2}{*}{ TM (\%) } & Control & $37.6 \pm 6.4^{A}$ & $8.4 \pm 1.9^{B}$ & $7.0 \pm 3.0^{B}$ & $0.6 \pm 0.2^{\mathrm{a}, \mathrm{B}}$ \\
\hline & WM & $27.6 \pm 3.1^{\mathrm{A}}$ & $8.0 \pm 2.3^{B}$ & $8.8 \pm 2.6^{B}$ & $5.8 \pm 1.2^{\mathrm{b}, \mathrm{B}}$ \\
\hline \multirow[t]{2}{*}{ PM (\%) } & Control & $20.8 \pm 3.9^{A}$ & $3.4 \pm 0.9^{B}$ & $3.0 \pm 1.4^{B}$ & $0.0 \pm 0.0^{\mathrm{a}, \mathrm{B}}$ \\
\hline & WM & $13.4 \pm 1.4^{\mathrm{A}}$ & $3.4 \pm 1.7^{B}$ & $3.4 \pm 0.8^{B}$ & $2.2 \pm 0.2^{\mathrm{b}, \mathrm{B}}$ \\
\hline \multirow[t]{2}{*}{$\operatorname{VAP}(\mu \mathrm{m} / \mathrm{s})$} & Control & $83.3 \pm 5.4^{A}$ & $67.4 \pm 7.6^{A B}$ & $53.9 \pm 5.0^{B}$ & $29.0 \pm 7.9^{\mathrm{a}, \mathrm{c}}$ \\
\hline & WM & $76.2 \pm 1.6$ & $54.7 \pm 5.7$ & $61.8 \pm 12.5$ & $53.5 \pm 3.2^{b}$ \\
\hline \multirow[t]{2}{*}{$\operatorname{VSL}(\mu \mathrm{m} / \mathrm{s})$} & Control & $61.6 \pm 4.2^{\mathrm{A}}$ & $50.5 \pm 9.2^{A B}$ & $36.0 \pm 4.5^{\mathrm{AB}}$ & $17.5 \pm 5.9^{\mathrm{a}, \mathrm{c}}$ \\
\hline & WM & $59.0 \pm 3.0$ & $40.3 \pm 7.0$ & $48.0 \pm 10.5$ & $42.0 \pm 3.4^{b}$ \\
\hline \multirow[t]{2}{*}{$\operatorname{VCL}(\mu \mathrm{m} / \mathrm{s})$} & Control & $144.6 \pm 11.2^{\mathrm{A}}$ & $109.4 \pm 11.0^{\mathrm{AB}}$ & $101.4 \pm 7.4^{B}$ & $70.1 \pm 19.1^{\mathrm{B}}$ \\
\hline & WM & $117.8 \pm 3.9^{A}$ & $88.3 \pm 4.5^{B}$ & $95.7 \pm 13.7^{A B}$ & $88.8 \pm 5.6^{B}$ \\
\hline \multirow[t]{2}{*}{ ALH $(\mu \mathrm{m})$} & Control & $7.7 \pm 0.5^{A}$ & $5.2 \pm 1.2^{\mathrm{AB}}$ & $6.5 \pm 0.5^{A}$ & $2.0 \pm 1.6^{a, B}$ \\
\hline & WM & $6.1 \pm 0.6$ & $4.8 \pm 0.5$ & $6.4 \pm 0.6$ & $6.4 \pm 0.7^{b}$ \\
\hline \multirow[t]{2}{*}{$\mathrm{BCF}(\mathrm{Hz})$} & Control & $29.5 \pm 1.6$ & $29.8 \pm 2.0$ & $32.2 \pm 2.5$ & $33.3 \pm 1.9$ \\
\hline & WM & $28.1 \pm 1.0$ & $30.1 \pm 1.4$ & $29.6 \pm 2.0$ & $26.0 \pm 1.4$ \\
\hline \multirow[t]{2}{*}{ STR (\%) } & Control & $68.6 \pm 1.9$ & $73.4 \pm 2.5$ & $67.0 \pm 3.5$ & $49.2 \pm 16.2$ \\
\hline & WM & $71.8 \pm 2.8$ & $70.4 \pm 5.2$ & $71.6 \pm 2.0$ & $78.4 \pm 2.5$ \\
\hline \multirow[t]{2}{*}{ LIN (\%) } & Control & $44.2 \pm 2.1^{\mathrm{A}}$ & $51.6 \pm 5.2^{A}$ & $42.6 \pm 3.2^{A}$ & $25.8 \pm 9.1^{\mathrm{a}, \mathrm{B}}$ \\
\hline & WM & $51.4 \pm 3.4$ & $50.6 \pm 4.9$ & $50.6 \pm 3.7$ & $55.8 \pm 2.3^{b}$ \\
\hline
\end{tabular}

a, bSmall letters indicate a significant difference between treatment groups in the same parameter $(p<0.05)$.

${ }^{A-C}$ Large letters indicate a significant difference with incubation time in the same treatment group $(p<0.05)$.

TM, total motility; PM, progressive motility; VAP, average path velocity; VSL, straight line velocity; VCL, curvilinear velocity; ALH, lateral head displacement; $\mathrm{BCF}$, beat cross frequency; STR, straightness; LIN, linearity.

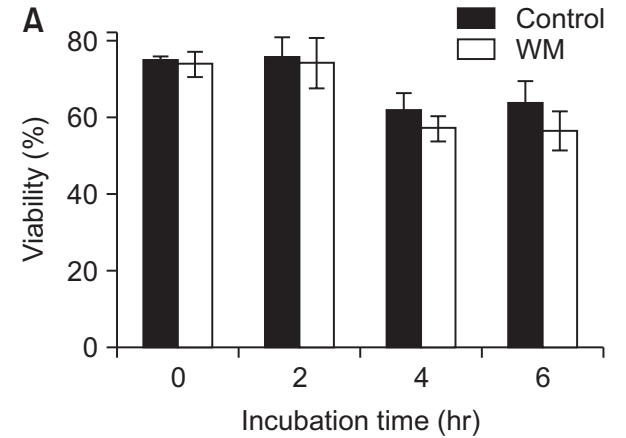

than the control group after $6 \mathrm{~h}$. The viability and acrosome reaction rates of sperm and the development rate of embryos were not affected by WM treatment.

The CASA procedure is a very useful technique for evaluating sperm motility and fertility. Although evaluating sperm motility using conventional microscopic methods is subjective, CASA can precisely analyze large numbers of spermatozoa in a short period. Assessment of sperm motility is efficient in predicting the potential fertility of sperm, as it plays an important role in the progression of sperm to the cervical mucus and penetration into the zona pellucida of oocytes (Verstegen et al., 2002). These motility and velocity parameters depend on factors such as collection time, age, temperature, $\mathrm{pH}$, and time between ejaculations (Wallach and Blasco, 1984).

Sperm motility has been used to predict fertility in livestock (Kang et al., 2020). In the present study, after $6 \mathrm{~h}$ of WM treatment PM and TM were significantly higher than in the control group, which showed a positive correlation with the velocity parameters VAP and VSL. Perumal et al. (2014) reported a similar result that during the same period, ALH and LIN of the WM treatment group showed 
higher values than those of the control group, and the effect of WM was not observed on VCL, BCF, and STR. Parameters such as PM, VAP, VSL, ALH, and BCF contribute to the total motility of spermatozoa and consequently correlate with sperm fertility (Kumar Yata et al., 2020). The VCL and ALH indicate hyperactivation of spermatozoa, and LIN indicates a measure of linearity, which is essential for fusion with oocytes (Perumal et al., 2014). Therefore, sperm motility was not inhibited by WM and could maintain motility longer than that of conventional sperm after a long period ( $\geq 6 \mathrm{~h}$ ). It could be expected that the process of separating sperm that is not aggregated with MNPs is similar to the swim-up method so that low-quality sperm could be filtered out.

Cryopreservation of semen increases the number of sperm exhibiting acrosome reaction and damage to the sperm membrane, which reduces viability and fertility (Felipe-Pérez et al., 2008; Almubarak et al., 2021). There is a need for an efficient approach to minimize damage to sperm during sex separation of frozen-thawed semen. Sperm capacitation and acrosome reactions are essential biochemical processes that allow sperm to penetrate the zona pellucida of oocytes (Breitbart, 2002). This study indicates that sex separation of sperm by WM treatment does not affect the proportion of viable and acrosomereacted sperm. Thongkham et al. (2021) reported that immunological sexing did not negatively affect sperm acrosome integrity but lowered the percentage of live sperm.

In general, frozen semen straw of Hanwoo contain 1.8 to $2.5 \times 10^{7}$ sperm. In artificial insemination (AI) in cattle, sperm numbers ranging from 0.5 to $1.5 \times 10^{7}$ sperm per insemination dose have been found to be optimal for fertility (Sullivan and Elliott, 1968). Sperm counts $0.5 \times$ $10^{6} \mathrm{sperm} / \mathrm{straw}$ and $5.0 \times 10^{6} \mathrm{sperm} / \mathrm{straw}$ were considered suboptimal for liquid and frozen semen, respectively (Mohanty et al., 2018). Also, a study on effects of two different sperm doses showed that fertilization rates were improved for both fresh and frozen sperm does, however, the percentages of motile to viable sperm was not affected by the increased sperm concentration (Nadir et al., 1993).

Barsuren et al. (2019) reported that sex-controlled calves were produced without a decrease in pregnancy rate and no increase or decrease in duration of pregnancy when using WM not combined with MNPs. According to a previous report, it was possible to successful sperm sexing and control of embryos using WM which MNPs were not combined (Heo et al., 2018). With the use of WM, the concentration of sperm will be halved due to sex segregation, and it is assumed that the reduced concentration would not affect fertility of sperm or pregnancy during AI. In addition, it is thought that the economic efficiency of farms could be improved through sex pre-select by applying WM to embryo transfer in cattle.

\section{CONCLUSION}

The monoclonal antibody conjugated with MNPs used for immunological sperm discrimination caused aggregation of Y sperm in response to WM treatment and did not negatively affect the characteristics of bull sperm. These results suggest that compared to other techniques, the immunological method for sex separation of sperm can classify sperm quickly and efficiently without use of expensive equipment.

Author Contributions: Conceptualization, D.K.K., C.K.P.; methodology, Y.H., S.H.L., H.T.C., C.K.P.; investigation, S.Y.J., Y.H.; data curation, S.Y.J.; writing-original draft preparation, S.Y.J.; writing-review and editing, supervision, S.Y.J., S.H.L., C.K.P.; administration, C.K.P.; funding acquisition, C.K.P.

Funding: This work was supported by Korea Institute of Planning and Evaluation for Technology in Food, Agriculture and Forestry (IPET) through Agri-food R\&D Performance Follow-up Support Program, funded by Ministry of Agriculture, Food and Rural Affairs (MAFRA) (120034-1).

Ethical Approval: All procedures that involved the use of animals were approved by the Kangwon National University Institutional Animal Care and Use Committee (KIACUC-09-0139).

Consent to Participate: All authors agree consent of participation.

Consent to Publish: All authors agree consent of publication.

Availability of Data and Materials: Upon reasonable request, the datasets of this study can be available from the 
corresponding author.

\section{Acknowledgements: None.}

Conflicts of Interest: No potential conflict of interest relevant to this article was reported.

\section{REFERENCES}

Al-Dulaimi SSH and Al-Timimi IHS. 2018. Separation of X spermatozoa from cauda of epididymis prior to selection of female embryos in local goat. Al-Anbar J. Vet. Sci. 11:84-94.

Almubarak AM, Kim W, Abdelbagi NH, Balla SE, Yu IJ, Jeon Y. 2021. Washing solution and centrifugation affect kinematics of cryopreserved boar semen. J. Anim. Reprod. Biotechnol. 36:69-75.

Barsuren E, Kim SH, Lee HJ, Yoon JT. 2019. Effect of embryo transfer seven days after artificial insemination with sexed and conventional semen from superovulated cattle. J. Anim. Reprod. Biotechnol. 34:106-110.

Bennett D and Boyse EA. 1973. Sex ratio in progeny of mice inseminated with sperm treated with $\mathrm{H}-\mathrm{Y}$ antiserum. Nature 246:308-309.

Breitbart H. 2002. Intracellular calcium regulation in sperm capacitation and acrosomal reaction. Mol. Cell. Endocrinol. 187:139-144.

Cui KH. 1997. Size differences between human X and Y spermatozoa and prefertilization diagnosis. Mol. Hum. Reprod. 3:61-67.

De Canio M, Soggiu A, Piras C, Bonizzi L, Galli A, Urbani A, Roncada P. 2014. Differential protein profile in sexed bovine semen: shotgun proteomics investigation. Mol. Biosyst. 10:1264-1271.

Felipe-Pérez YE, Juárez-Mosqueda ML, Hernández-González EO, Valencia JJ. 2008. Viability of fresh and frozen bull sperm compared by two staining techniques. Acta Vet. Bras. 2:123130.

Garner DL, Gledhill BL, Pinkel D, Lake S, Stephenson D, Van Dilla MA, Johnson LA. 1983. Quantification of the X- and Ychromosome-bearing spermatozoa of domestic animals by flow cytometry. Biol. Reprod. 28:312-321.

Han TL, Flaherty SP, Ford JH, Matthews CD. 1993. Detection of $\mathrm{X}$ - and Y-bearing human spermatozoa after motile sperm isolation by swim-up. Fertil. Steril. 60:1046-1051.

Heo YT, Kim DG, Uhm S. 2018. Analysis of sex ratio on bovine in vitro fertilized embryos using sex determination kit treated sperm. J. Emb. Trans. 33:169-175.

Johnson LA and Clarke RN. 1988. Flow sorting of X and Y chromosome-bearing mammalian sperm: activation and pronuclear development of sorted bull, boar, and ram sperm microinjected into hamster oocytes. Gamete Res. 21:335343.

Kaneko S, Iizuka R, Oshiro S, Nakajima H, Oshio S, Mohri H.
1983. Separation of human X- and Y-bearing sperm using free-flow electrophoresis. Proc. Jpn. Acad. Ser. B Phys. Biol. Sci. 59:276-279.

Kang SS, Kim UH, Lee MS, Lee SD, Cho SR. 2020. Spermatozoa motility, viability, acrosome integrity, mitochondrial membrane potential and plasma membrane integrity in $0.25 \mathrm{~mL}$ and $0.5 \mathrm{~mL}$ straw after frozen-thawing in Hanwoo bull. J. Anim. Reprod. Biotechnol. 35:307-314.

Koundouros S and Verma P. 2012. Significant enrichment of Ybearing chromosome human spermatozoa using a modified centrifugation technique. Int. J. Androl. 35:880-886.

Kumar Yata V, Kumar Gangwar D, Sharma V, Kumar Dubey S, Kumar Yadav S, Choudhary S, Kumar S, Kumar Mohanty T, Kumar Mohanty A. 2020. Semen analysis and sperm characteristics of Karan Fries cattle. Anim. Reprod. Sci. 212:106250.

Laxmivandana R, Patole C, Sharma TR, Sharma KK, Naskar S. 2021. Differential proteins associated with plasma membrane in X- and/or Y-chromosome bearing spermatozoa in indicus cattle. Reprod. Domest. Anim. 56:928-935.

Li CJ, Wang D, Zhou X. 2016. Sperm proteome and reproductive technologies in mammals. Anim. Reprod. Sci. 173:1-7.

Mohanty TK, Lone SA, Kumaresan A, Bhakat M, Kumar R, Baithalu RK, Sinha R, Paray AR, Yadav HP, Sahu SK, Mohanty AK. 2018. Sperm dosage and site of insemination in relation to fertility in bovines. Asian Pac. J. Reprod. 7:1-5.

Nadir S, Saacke RG, Bame J, Mullins J, Degelos S. 1993. Effect of freezing semen and dosage of sperm on number of accessory sperm, fertility, and embryo quality in artificially inseminated cattle. J. Anim. Sci. 71:199-204.

Penfold LM, Holt C, Holt WV, Welch GR, Cran DG, Johnson LA. 1998. Comparative motility of $\mathrm{X}$ and $\mathrm{Y}$ chromosome-bearing bovine sperm separated on the basis of DNA content by flow sorting. Mol. Reprod. Dev. 50:323-327.

Perumal P, Srivastava SK, Ghosh S, Baruah KK. 2014. Computer-assisted sperm analysis of freezable and nonfreezable Mithun (Bos frontalis) semen. J. Anim. 2014:675031.

Prasad S, Rangasamy S, Satheshkumar S. 2010. Sex preselection in domestic animals - current status and future prospects. Vet. World 3:346-348.

Safari A, Ghavi Hossein-Zadeh N, Shadparvar AA, Abdollahi Arpanahi R. 2018. A review on breeding and genetic strategies in Iranian buffaloes (Bubalus bubalis). Trop. Anim. Health Prod. 50:707-714.

Seidel GE Jr. 2012. Sexing mammalian sperm - where do we go from here? J. Reprod. Dev. 58:505-509.

Sullivan JJ and Elliott FI. 1968. Bull fertility as affected by an interaction between motile spermatozoa concentration and fertility level in artificial insemination. Proc. Int. Congr. Anim. Reprod. A. I. 2:1307-1310.

Thongkham M, Thaworn W, Pattanawong W, Teepatimakorn S, Mekchay S, Sringarm K. 2021. Spermatological parameters of immunologically sexed bull semen assessed by imaging flow cytometry, and dairy farm trial. Reprod. Biol. 21:100486.

Verstegen J, Iguer-Ouada M, Onclin K. 2002. Computer assisted 
semen analyzers in andrology research and veterinary practice. Theriogenology 57:149-179.

Wallach EE and Blasco L. 1984. Clinical tests of sperm fertilizing ability. Fertil. Steril. 41:177-192.
Zavos PM. 1983. Preconception sex determination via intravaginal administration of $\mathrm{H}-\mathrm{Y}$ antisera in rabbits. Theriogenology 20:235-240. 